Supplement of Saf. Nucl. Waste Disposal, 1, 261-262, 2021

https://doi.org/10.5194/sand-1-261-2021-supplement

(C) Author(s) 2021. CC BY 4.0 License.

Supplement of

\title{
Assessment of Partitioning and Transmutation of High-Level Waste and Hypothetical Implementation Scenarios in Germany
}

Friederike Frieß et al.

Correspondence to: Friederike Frieß (friederike.friess@boku.ac.at)

The copyright of individual parts of the supplement might differ from the article licence. 
Assessment of Partitioning and Transmutation of High Level Waste Hypothetical Implementation Scenarios in Germany

BOKU Wien, ISR

Friederike Frieß, Wolfgang Liebert

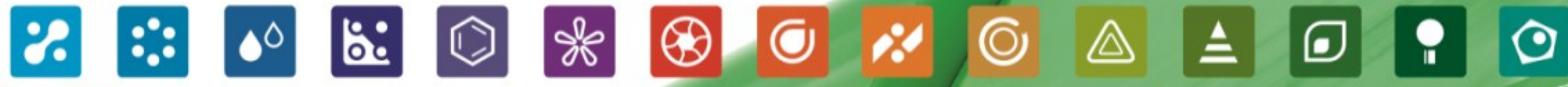




\section{Overview}

What is Partitioning \& Transmutation?

- Fuel Cycle

- Criteria for Assessment

- Technology

Simulation

- Scenarios + Assumptions

- Separated Plutonium Stockpile

- Minor Actinide Stockpiles

Conclusion 


\section{Partitioning and Transmutation (P\&T)}

Partitioning: Separation of spent nuclear fuel into different material streams

- (short-lived) fission products

- Plutonium

- Minor Actinides (MA): Np, Am, Cm

Transmutation: Transformation of long-lived radionuclides into short-lived or stable isotopes

Goal: Significantly reduced requirements on long-term radioactive waste management 


\section{P\&T Fuel Cycle}

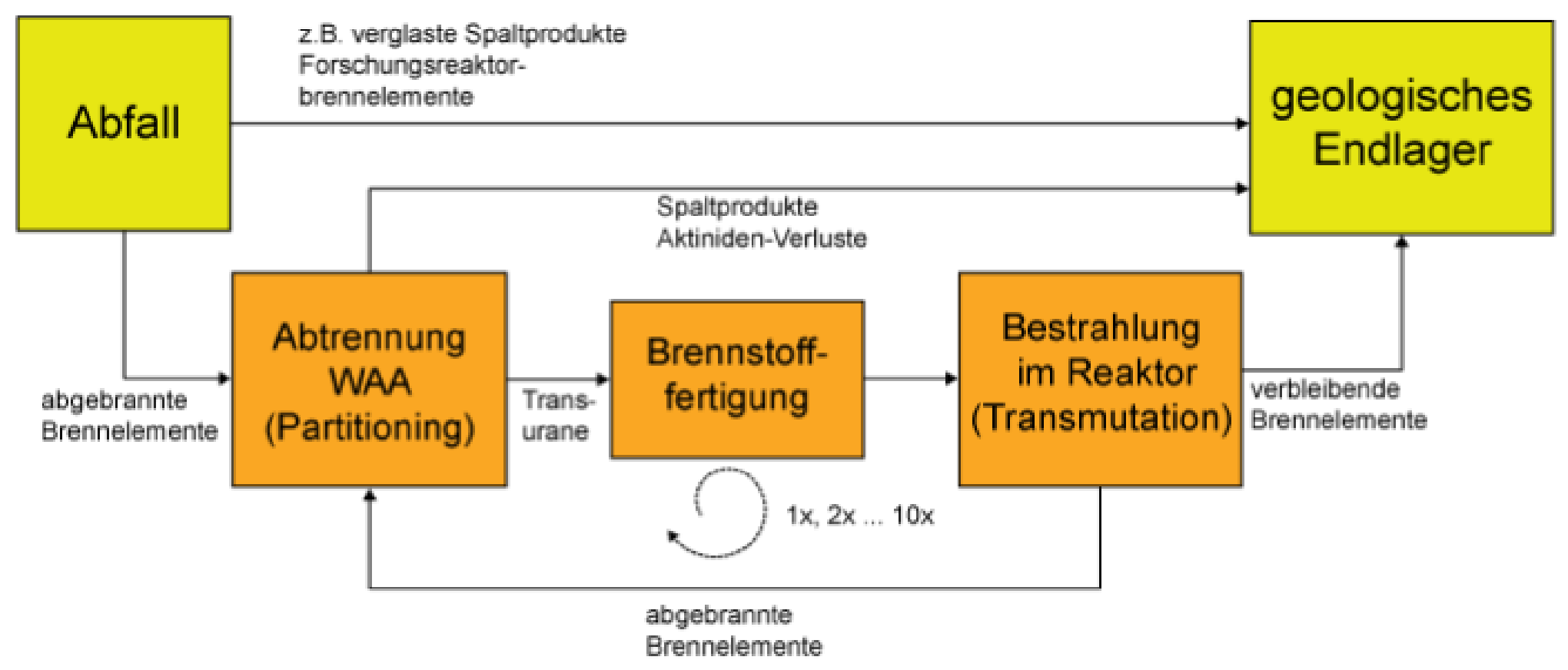

Source: Öko-Institut e.V. 


\section{Criteria for P\&T Assessment}

- Technology availability (needed R\&D, development risks)

- Risks

- Safety (new reactor types, various fuel compositions, regulations...)

- Security (proliferation risks by access to weapons-usable materials)

- Safeguards (new measures needed)

- Implementation period

- Effect on radioactive waste disposal (radiotoxicity vs. long-term safety, decay heat, volume)

- Overall energy production strategy (phase-out?) 


\section{Choices of Technology in P\&T Fuel Cycles}

Not available on industrial scale:

Reprocessing:

- Pyroprocessing

- Hydroprocessing (e.g. PUREX)

Fuel:

- Mixed-Oxide Fuel (MOX) with increased MA content

- Inert-Matrix fuel (IMF) - no Uranium

- Molten salt

Irradiation facility:

- Critical Fast Reactors (FR)

- Accelerator-driven systems (ADS)

- Molten-salt Reactors (MSR) 


\section{Modeling Goals and Challenge}

P\&T requires 5-10 irradiation cycles, depending on implementation path

$\Rightarrow$ analysis of different hypothetical scenarios

- How long would the scenario last?

- How many and what kind of facilities would be needed?

- What are the actual effects on waste streams \& disposal requirements?

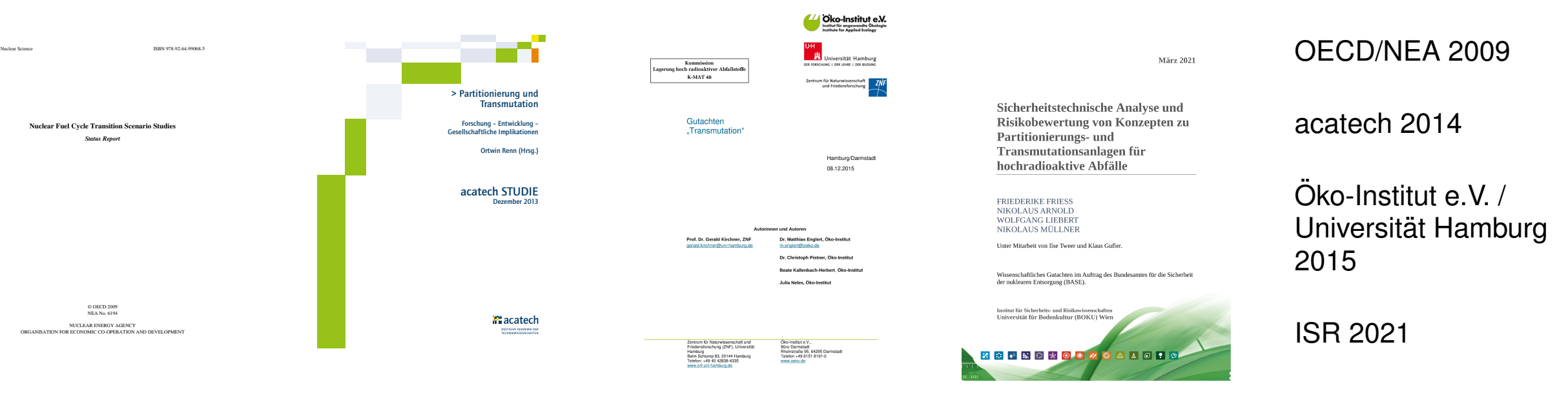




\section{Scenarios}

Scenario 1: FR (based on ASTRID)

- Hydrochemical reprocessing

- MOX fuel (MA enriched)

Scenario 2: ADS (based on EFIT)

- Inert matrix fuel (IMF)

- Pyrochemical reprocessing

Scenario 3: MSR (based on MOSART)

- Molten salt

- Pyroprocessing

Sources:GIF; www.bellona.org; www.duboichemicals.com 


\section{Fuel Composition in P\&T Fuel Cycle}

(U), Pu, MA fractions change with each irradiation cycle

$\Rightarrow$ Influence on reactor dynamics?

$\Rightarrow$ Adaption of reactor design?

Our assumption: element fraction constant - no adaption needed

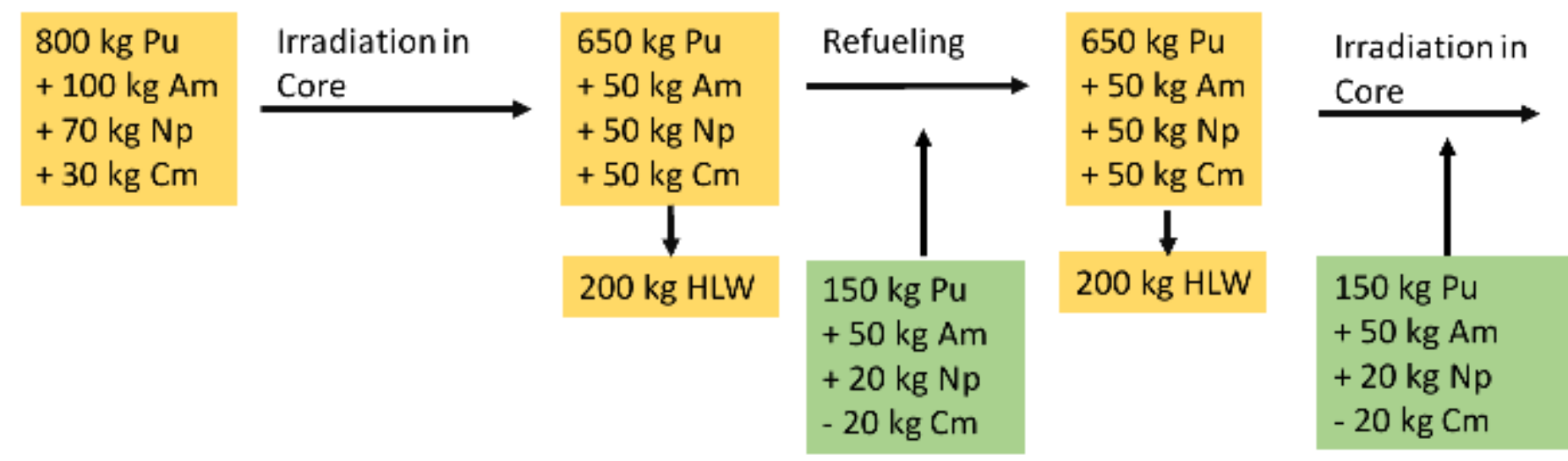

Plutonium, Neptunium, Americium, and Curium Stockpile - which one is lacking in the end? 


\section{Model Assumptions}

- All necessary technology available on industrial scale

- P\&T only suitable for spent fuel elements, not for vitrified waste

- Transmutation efficiencies per element, not per isotope

- No detailed modeling of fission products (estimate based on cross sections)

- Facilities are operated total lifetime

- Radioactive decay / Uranium inventory is not considered 


\section{Starting Situation - German Inventory}

\begin{tabular}{lrr}
\hline Element (group) & Fraction & Inventory 2022 \\
\hline Total & $100.00 \%$ & $10,113.0 \mathrm{t}$ \\
Uranium & $94.27 \%$ & $9,534.0 \mathrm{t}$ \\
Plutonium & $1.27 \%$ & $128.6 \mathrm{t}$ \\
Minor Actinides (Np, Am, Cm) & $0.21 \%$ & $21.1 \mathrm{t}$ \\
Fission Products & $4.04 \%$ & $408.1 \mathrm{t}$ \\
\hline
\end{tabular}

Inventory 2022 estimated based on data from 2020. 


\section{Scenarios \& Facilities}

Scenario 1: FR (based on ASTRID, 1200 MWth)

- max 8 facilities, 23 in total

- cycle length 11 years (hydroprocessing)

- total scenario length $\approx 300$ years

Scenario 2: ADS (based on EFIT, 400 MWth)

- 2 facilities, consecutively

- cycle length 7 years

- total scenario length $\approx 90$ years

Scenario 3: MSR (based on MOSART, 2400 MWth)

- 3 facilities at the same time

- cycle length 1 year

- total scenario length $\approx 55$ years 


\section{Separated Plutonium Stockpile - FR}

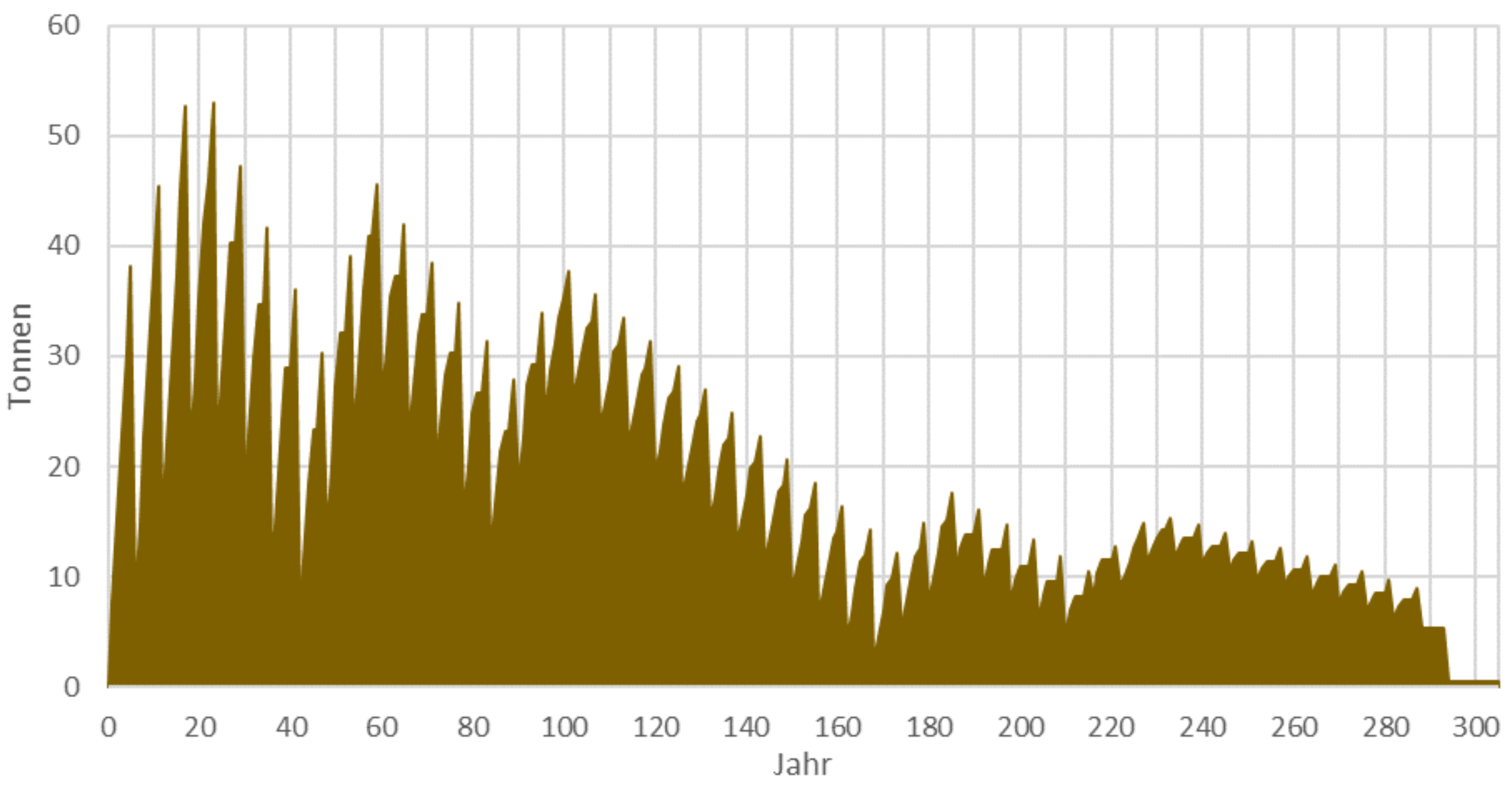




\section{Stockpile of Transuranium Elements - FR}

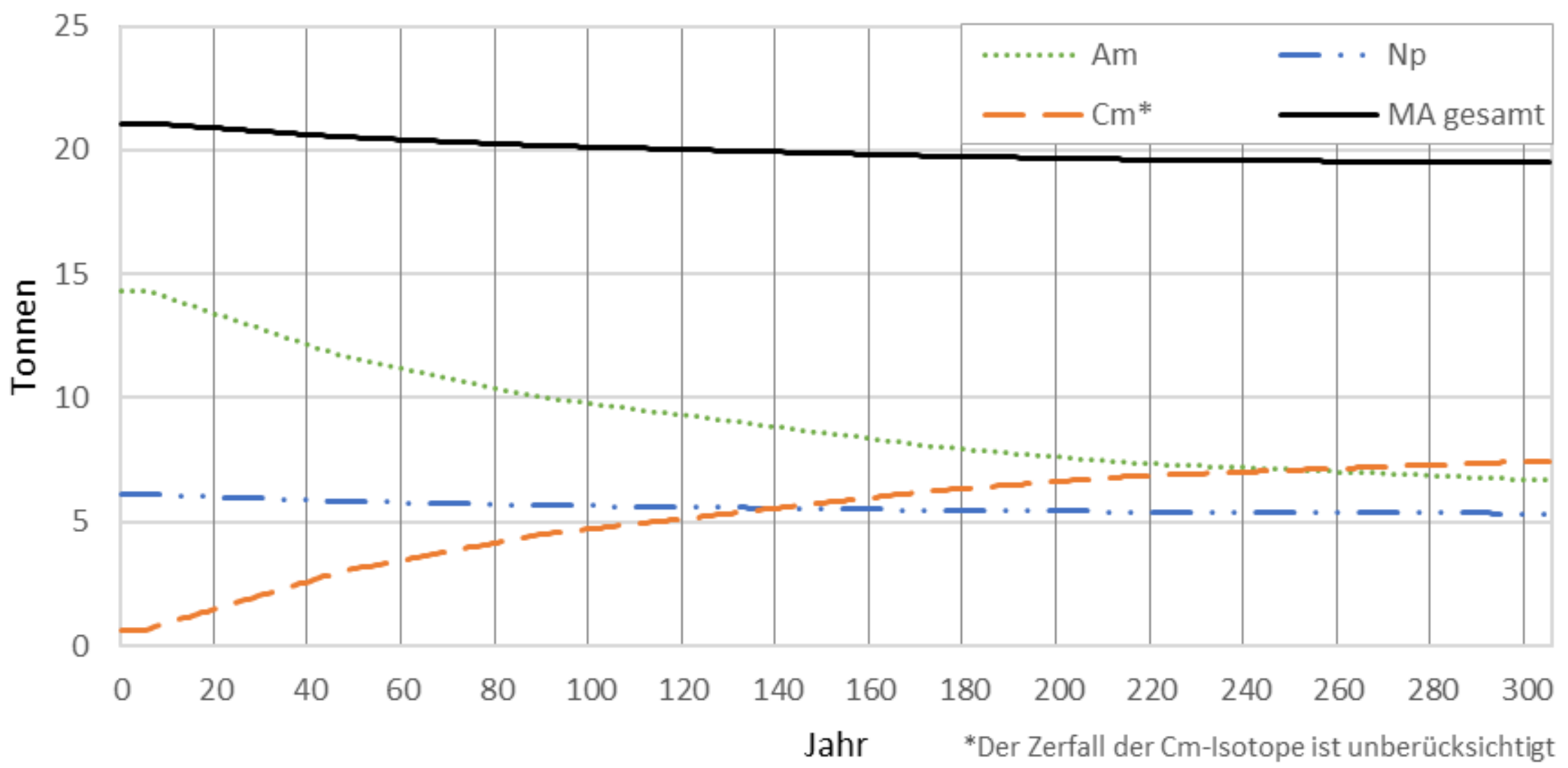




\section{Separated Plutonium Stockpile - ADS}

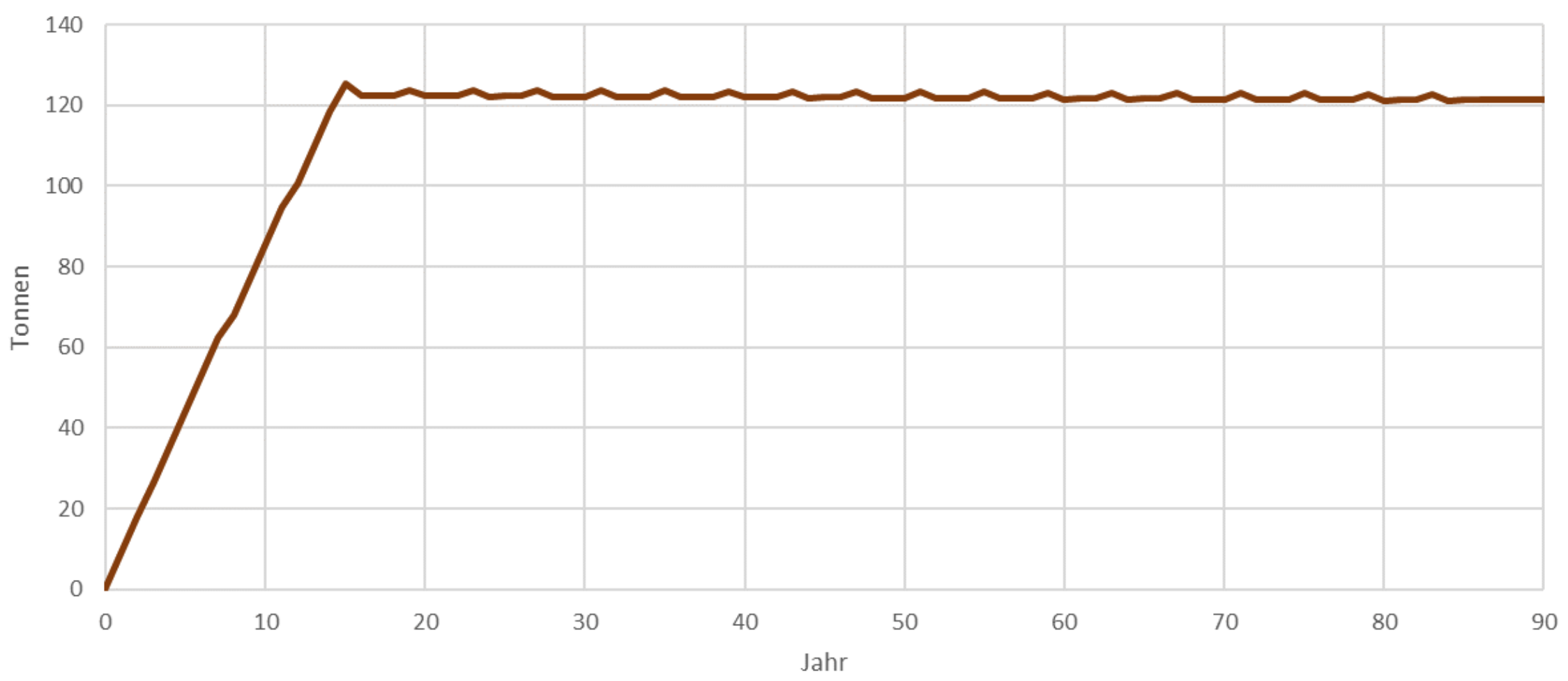




\section{Stockpile of Transuranium Elements - ADS}

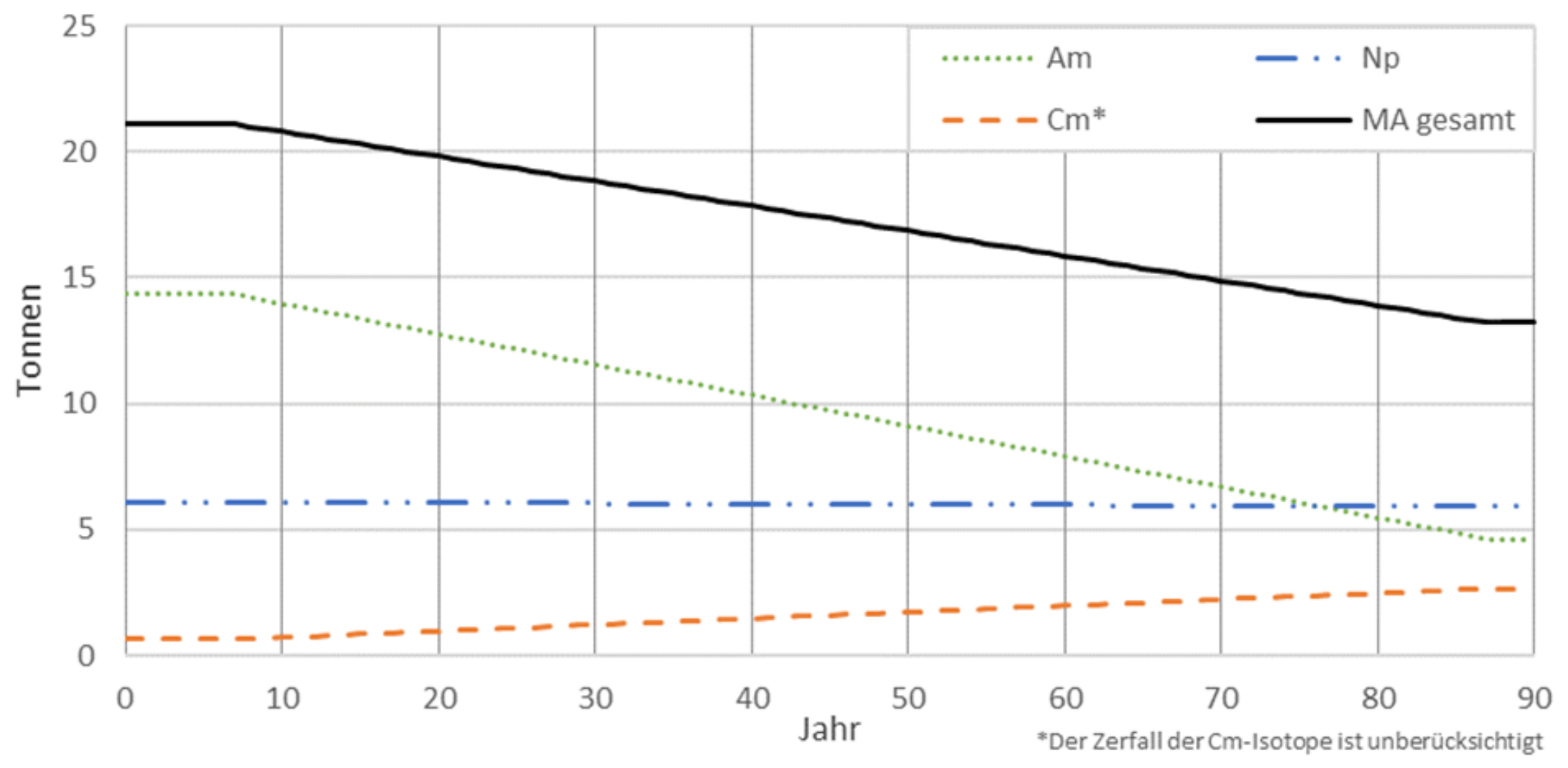




\section{Separated Plutonium Stockpile - MSR}

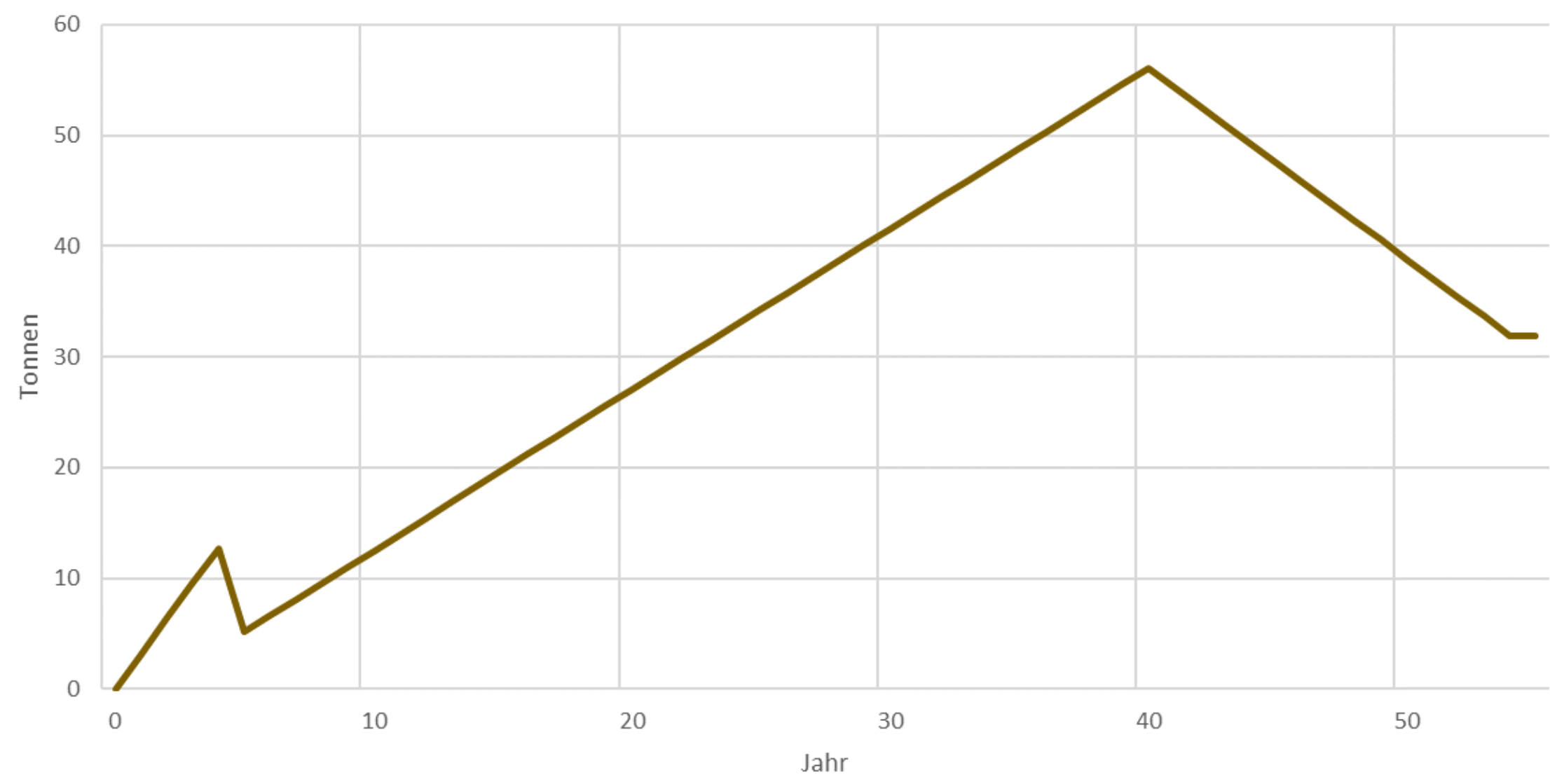




\section{Stockpile of Transuranium Elements - MSR}

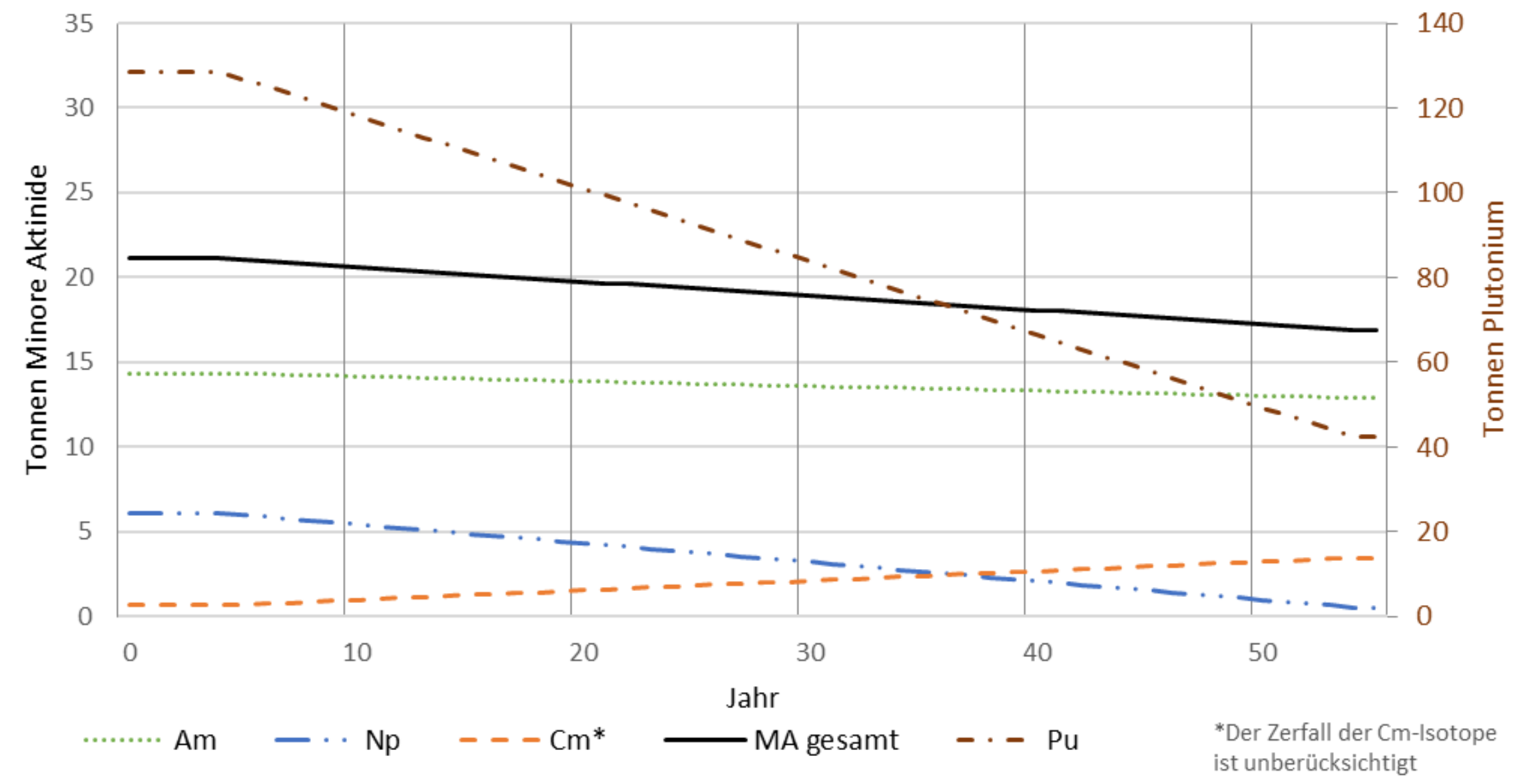




\section{Additional Waste Streams}

\begin{tabular}{lrrr}
\hline & FR & ADS & MSR \\
\hline LLFP - Tc-99 & $+48 \%$ & $+3 \%$ & $+23 \%$ \\
LLFP - I-129 & $+63 \%$ & $+3 \%$ & $+27 \%$ \\
LLFP - Cs-135 & $+71 \%$ & $+9 \%$ & $+76 \%$ \\
HLW & $2,300 \mathrm{~m}^{3}$ & $7,400 \mathrm{~m}^{3}$ & $7,350 \mathrm{~m}^{3}$ \\
Long-lived Waste & $84,000 \mathrm{~m}^{3}$ & $23,000 \mathrm{~m}^{3}$ & $23,000 \mathrm{~m}^{3}$ \\
Dismantling & $323,000 \mathrm{~m}^{3}$ & unknown & unkown \\
\hline
\end{tabular}

LLFP: Long-lived Fission Products HLW: High Level Waste

Konrad Repository: 300,000 m³ 


\section{Conclusion I - Simulation Results}

- P\&T requires long-term operation of several advanced nuclear facilities

- Amount of low and intermediate level waste (LILW) increases significantly

- Stockpiles of long-lived fission products increase substantially

- high level waste (HLW) repository is needed in any case

- Separated fissile material, especially Plutonium is present / left

- Unresolved safety issues (changing fuel composition) 


\section{Conclusion II}

- Technology not ready (decades of R\&D)

- Reactor models fit to German situation are lacking

- Model assumptions of uttermost importance

$\Rightarrow$ Significant impact on results

$\Rightarrow$ more data needed

- P\&T scenarios postpone final disposal of HLW / closing of deep geological repository

Following a P\&T path would be a nuclear program for many decades (or even centuries). 


\section{Thank you}

Frieß, F., Arnold, N., Liebert, W., and Müllner, N.: Sicherheitstechnische Analyse und Risikobewertung von Konzepten zu Partitionierungs- und Transmutationsanlagen für hochradioaktive Abfälle, Wissenschaftliches Gutachten im Auftrag des Bundesamtes für die Sicherheit der nuklearen Entsorgung (BASE), Berlin, 280 pp.,urn:nbn:de:0221-2021030826033, März 2021.

The report includes an English summary. 
University of Natural Resources and Life Sciences, Vienna Institute for Safety and Risk Sciences

Friederike Frieß, Wolfgang Liebert

Dänenstraße 4, A-1190 Wien

Tel: +43 1 47654-81821

Mail: friederike.friess@boku.ac.at www.boku.ac.at

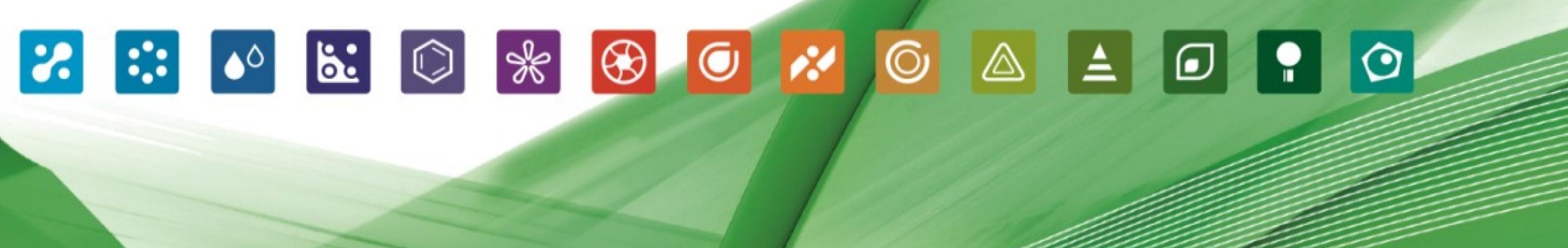

